\title{
Welche Mindestforderungen sind an Malz für Malzkaffee zu stellen?
}

Von

Heinrich Trillich in Karlsruhe-Rüppurr.

In den deutschen „Vereinbarungen“ findet sich hinsichtlich der Kaffeesurrogate der Grundsatz, dal sie unter einer ihrer wirklichen Beschaffenheit entsprechenden Bezeichnung in den Handel zu bringen sind ${ }^{1}$ ); es müssen also als "Malzkaffee" bezeichnete Produkte sirngemäß aus Malz hergestellt sein.

Wer jedoch in die Lage gekommen ist, die sehr zablreichen als „Malzkaffee“ gehandelten Produkte fortlaufend und näher zu untersuchen, wird über ihre Verschiedenheit sehr erstaunt gewesen sein. Nicht nur, dab gegen frủher, wo man nur trocken geröstetes Brenn- und Braumalz, das sog. Farbmalz, kannte, seit 1890 die von mir eingeführte Röstung durchfeuchteter Malze - nach Analogie der sog. Karamelmalze - wesentlich andere, besser schmeckende Malzkaffees ergab, es zeigte sich auch sehr bald eine große Unsicherheit über den Begriff, „Malz" 2) selbst und diese Unsicherheit ist nicht nur bis jetzt nicht gewichen, sie ist geradezu größer geworden.

Die Tatsache, daß rohe wie gemälzte Cerealien durchaus andere Produkte geben, wenn sie im durchweichten Zustande sofort geröstet werden, gegenüber denen, die durch Rösten eines trockenen Getreides oder eines völlig trocken gedarrten Malzes erhalten werden, ist in ihrer Tragweite in praktischer und analytischer Beziehung nicht genügend bekannt.

Trcckene Röstung gibt dunkle, bitter und scharf schmeckende Röstprodukte mit Extraktziffern von $60-65 \%$, einen mehligen Bruch, grauviolette Farbe mit Milch wir haben es im wesentlichen mit stark dextrinisierter Stärke, bezw. Röstbitter zu tun, beim Malz ist der Zucker schon überkaramelisiert.

Feuchte Röstung gibt hellere, relativ mild schmeckende Röstprodukte mit Extraktziffern von $30-40 \%$, einen glasig krystallinischen Brush, kaffeebraune Farbe mit Milch - wir haben es im wesentlichen mit einem vor dem Rösten verkleisterten Korninhalt zu tun, der sich bei relativ niederer Temperatur bräunt und helle Maltosekaramele enthält.

Obwohl ich schon früher diese Verhältnisse geschildert habe ${ }^{3}$ ), mul ich sie doch berühren, weil der niedere Extraktgehalt solcher feucht gerösteter Produkte zu mebreren Mißdeutungen geführt hat, so z. B. zur Behauptung einer „schwachen“ Röstung - oder einer "Auslaugung der Maltose“ oder endlich einer zu schwachen Mälzung. Diese letztere findet sich auch in der neuen Auflage der „Chemie der menschlichen Nahrungs- und Genußmittel $\left.{ }^{4}\right)^{\text {“ }}$ von König.

1) Forschungsberichte über Lebensmittel etc. 1894, 1, 411; 1895, 2, 275; 1896, 3, 351 und "Vereinbarungen“ 1902, Heft III, S. 37.

2) Forschungsberichte über Lebensmittel etc. 1896, 3, 351 .

3) Zeitschr. angew. Chem. 1891, 540 und 719.

4) J. König, Chemie der menschlichen Nahrungs- und Genufmittel. Berlin, Julius Springer. 4. Aufl., 1904, 2, 1093. 
König sagt: „Da gutes Malz 70-80\% Extraktausbeute liefert, so kann ein Malzkaffee „unter Berücksichtigung des Röstverlustes 55-70\% Wasserextrakt liefern. Größere Abweichungen von der unteren oder oberen Grenze müssen entweder auf die geringere oder größere Keimung (Mälzen) oder auf die stärkere oder schwächere Röstung zurückgeführt werden."

Ich möchte dazu bemerken, daß auch das beste Malz nur dann $70-80 \%$ Extrakt liefert, wenn es braumäBig vermaischt wird — sowie man aber die von mir vorgeschlagene Extraktbestimmung, die der praktischen Kaffeebereitung nachgebildet ist, anwendet, scheidet natürlich die maischende Wirkung der Diastase aus und man erhält nur 45-55 \% Extrakt - beim Malzkaffee hat man überbaupt keine Diastase mehr. Es sind also falsche Größen in Vergleich gesetzt. Ganz abgesehen davon ist aber lediglich die Verkleisterung bei der feuchten Röstung die Ursache der geringeren Extraktzahl, aber auch des besseren Geschmackes.

Daß die Extraktziffer an sich für den Genußmittelwert ohne Bedeutung ist, habe ich schon wiederholt betont; es berührt mich immer peinlich, wenn ich lese, der Malzkaffe A mit vielleicht $50 \%$ Extrakt sei dem Malzkaffee B mit nur $35 \%$ seines höheren Extraktgehaltes und Nährwertes wegen vorzuzieben. Genau mit demselben Recht kann man sagen, Zichorie mit $70 \%$ Extrakt ist dem feinsten Bohnenkaffee mit nur $25 \%$ Extrakt dreimal vorzuziehen. Ausschlaggebend ist eigentlich nur der chemisch nicht faßbare Genufmittelwert, denn tataächlich wird der feucht geröstete Malzkaffee mit 35-45\% Extrakt überall dem Farbmalz mit $60-65 \%$ Extrakt vorgezogen, und genau so liegen die Verhältnisse bei feucht und trocken gerösteter Gerste bezw. anderen Cerealien.

Die äußerliche Ähnlichkeit zwischen feucht geröstetem $\mathrm{Malz}$ und feucht gerösteter Gerste, die sich für den Nichtpraktiker auch auf den Schnitt erstreckt, dann die naheliegenden Gedanken, weder Karamelmalz auf Malzkaffee weiterzurösten, noch Darrmalz neuerdings einzuweichen, sondern direkt feuchtes Grünmalz zu rösten, waren aber Ursache, daß zahlreiche Fabrikate auftauchten, die zur Führung des Namens „Malzkaffee“ wohl nicht berechtigt sind.

Selbst diejenigen Fabrikanten, die sich die Mälzungsanlage, die Mälzungskosten und die Mälzungsverluste gänzlich sparten, einfach Gerste einweichten und dann brannten, fanden Verteidiger für ihre Behauptung, das sei ebenfalls Malz - ja vor einem Berliner Gericht erfolgte Freisprechung, weil man unglaublicherweise sogar von einem „Zichorienmalz", das sind genäßte Zichorienschnitzel, sprach. Nun ist uns Süddeutschen der Begriff „Malz" allerdings mehr ins Blut übergegangen, als den Norddeutschen, aber daß man einen bloßen Einweichprozeß als Mälzung bezeichnet, ist doch kaum glaublich.

Trotzdem ist in einem neuerlichen Prozeß von einem Herrn des Berliner Instituts für Gärungsgewerbe die Behauptung aufgestellt worden - „man könnte unter Umständen schon geweichte Gerste als Malz bezeichnen". Diese der bisherigen Auffassung des Begriffes "Malz und Mälzen“ widersprechende Ansicht wird nach meinen Erkundigungen mit der Möglichkeit begründet, daß schon beim Einweichen eine Enzymogenbildung, d. b. die Bildung derjenigen Fermente beginnt, die die Eiweißkörper in Enzym, d. i. Diastase umwandeln.

Nach meinem Dafürhalten haben wir keine Ursache, diese vorerst rein hypothetische Auffassung uns zu eigen zu machen, denn wenn man geweichte Gerste darrt, 
wird sie niemand als Malz kaufen - erst das Produkt, in dem das Enzym Diastase entwickelt ist und schon kräftig gewirkt hat, ist praktisch „Malz“.

Die meisten neueren Versuche, Gerstenkaffee als Malzkaffee zu verkaufen, haben zu Verurteilungen meist auf Grund des $\S 1$ des Gesetzes über unlauteren Wettbewerb geführt, indem Anpreisung und Benennung etwas Wertvolleres voraussetzen lassen (Malz), als tatsächlich geliefert wird (Gerste).

Auch gegen die Verwendung der Bezeichnung "Malzgerstenkaffee" muß ich mich aussprechen, da sie eine Verschleierung bezweckt und nicht ohne weiteres auf einen aus „Malzgerste" hergestellten Gerstenkaffee schließen läßt, wohei die mälzungsfähige Gerste in Gegensatz zu Futtergerste treten soll, aber von vornherein ihren Beruf verfehlt, weil sie ja gar nicht gemälzt wird.

Nun gibt es aber von der bloß in Wasser eingeweichten Gerste bis zum fertigen Darrmalz zahlreiche Zwischenstufen, sog. Spitzgerste, Grünmalz mit seinen verschiedenen Entwickelungsstufen, Schwelkmalz, Darrmalz, und sehr bald trat die Frage auf, von welchem Stadium an kann eine keimende Gerste eigentlich als Malz bezeichnet werden?

Für die Brauerei, Brennerei und Malzextraktfabrikation konnte eine solche Frage eigentlich nicht auftauchen, weil man aus alter Praxis ganz bestimmte Wachstumseigenschaften $\mathrm{zu}$ fordern gewohnt ist, aber findige Malzkaffeefabrikanten machten einfach den Einwand, ja so gut man für alle anderen Zwecke besondere Malze darstelle, so gut müßten sie für Malzkaffee eben ein besonders kurz entwickeltes Malz herstellen und wenn sie dann nicht an der Quellgerste hängen blieben, so war es sicher bei der Spitzgerste. Ich hatte schon 1896 darauf aufmerksam gemacht, daß man allerdings nicht verlangen kann, daß Malzkaffee aus Brauereimalz bergestellt seì, weil dieser einen süßen Geschmack hat, der nur in Gegenden mit Feigenkaffeekonsum beliebt ist, während Gegenden mit Zichorienkonsum einen kräftigen Geschmack wollen, aber trotzdem soll Malz eben doch Malz sein.

Jch habe zahlreiche Malzkaffees im Handel gefunden, die sicher keinen eigentlichen Wachstumsprozeß durchgemacht haben, denn sie zeigten noch keinerlei oder nur unbedeutende Blattkeimentwickelung.

Gerade diese relativ leicht festzustellende Blattkeimentwickelung ist beim Malzkaffee vorläufig das einzige Beweismittel, daß überhaupt eine Keimung stattgefunden hat und wieweit diese Keimung ungefähr getrieben war.

Ich kann mich mit der in manchen Lehrbüchern stehenden Definition, „Malz ist geweichte und gekeimte Gerste, deren Keimungsprozeß unterbrochen wurde", in dieser unbeschränkten Fassung nicht einverstanden erklären; die sämtlichen Gewerbe, die Malz machen und Malz handeln, präzisieren den Keimungsprozeß erst von der Bildung einer überschüssigen Diastasemenge und zugleich „Auflösung“ des Korninhalts an, wobei rund die Hälfte der Kohlenhydrate löslich wird.

Gleichgültig ob es sich im späteren Verlauf des. Mälzens um Herstellung von Braumalz und Brennereimalz oder Kaffeemalz handelt, stets wird man von Malz nur reden können, wenn das "Auflösungs"-Stadium durchschritten worden ist.

Damit ist aber stets eine relative Entwickelung des Blattkeims bis mindestens zur halben Kornlänge verknüpft und ich habe den maßgebenderen Malzkaffeefabrikanten im Interesse einer bestimmten Begriffsfestlegung vorgeschlagen, als Malzkaffee nur Produkte herzustellen und anzuerkennen, bei denen der Mälzungsprozeß durch einen Blattkeim von mindestens halber Kornlänge dokumentiert ist. Natürlich muß 
man die natürlichen Verschiedenheiten in der Keimenergie dabei berücksichtigen, wenigstens 100 Körner untersuchen, aber die Festlegung einer Art Minimalbegriff ist unbedingt notwendig. Die Fabrikate der meisten und gerade der größeren Fabriken zeigen sich durchaus weiter ausgemälzt, als damit gefordert ist, sodaß von einer Erschwerung ganz gewiß nicht gesprochen werden kann.

Wie schon erwähnt, versagen alle anderen Nachweise einer Mälzung, die diastatische Kraft ist zerstört, die Maltose ist karamelisiert, die Maltodextrine und die Stärke sind dextrinisiert, ja meistens darüber hinaus abgebaut - das Reduktionsverhältnis für Fehling'sche Lösung ist stark schwankend - die sonstigen Unterschiede im Schnitt, im Geschmack, in der Filtrationsfähigkeit etc. kennt nur der Praktiker die Wurzelkeime sind abgefallen. Die Forderung einer durchschnittlichen Blattkeimentwickelung von halber Kornlänge ist tatsächlich eine Minimalanforderung an Malz, wenn man darunter einigermaßen das Produkt verstehen will, das bisher die Technik and die Wissenschaft, wie auch der Handel als "Malz" im allgemeinen bezeichnet haben und das, wie schon bemerkt, auch von der überwiegenden Mehrzahl der Malzkaffeefabrikanten als Ausgangspunkt benutzt wird.

Auch der Konsument hat ein Interesse an dieser Minimalforderung, denn er muß für Malzkaffee einen durch Einrichtungen, Arbeit und Substanzverluste bedingten höheren Preis anlegen; erhält er dann Gerstenkaffee, so ist er eben benachteiligt, denn Gerstenkaffee kann er billiger kaufen; wird ein solches Produkt aber als Malzkaffee billiger verkauft, so erweckt es den Eindruck eines vorteilhafteren Angebotes, in dem etwas Minderwertigeres geliefert wird, als die Bezeichnung erwarten läßt.

Da unsere Vereinigung ohnehin an eine Durchsicht der "Vereinbarungen" herangetreten ist, unterlasse ich es, jetzt schon bestimmte Anträge zu stellen, hoffe aber, dab meine Ausführungen zu einer einheitlichen Beurteilung auf diesem nicht unwichtigen Spezialgebiet beitragen.

\title{
Zur Beurteilung des Weinessigs.
}

\author{
Von
}

\section{W. Fresenius-Wiesbaden.}

M. H.! Die kurzen Mitteilungen, die ich Ihnen machen möchte, sind angeregt durch einige praktische Fälle aus meiner letzten Erfahrung. Mir sind wiederholt Gutachten vorgelegt worden, in welchen Weinessige lediglich deshalb beanstandet worden sind, weil ihr Glyceringehalt ein zu geringer war und bei denen deshalb nicht nur der Vorwurf erhoben wurde, daß sie nicht $20 \%$ Wein enthielten, sondern auch, daß sie im übrigen, durch Zusatz von Extrakt bezw. Mineralstoffen dem Weinessig täuschend nachgemacht seien.

$\mathrm{Da}$ ich meines Wissens der erste gewesen bin, der die Heranziehung der Glycerinbestimmung zur Entscheidung der Frage, ob Weinessig oder Spritessig vorliege, angeregt hat, so gestatten Sie mir wohl heute einmal den Stand unserer Erfabrungen über diese Frage, soweit ich sie habe verfolgen können, darzulegen und daran eine kurze Erörterung darüber anzuknüpfen, wie sichere und bestimmte Schlüsse man deshalb heute auf die Resultate der Glycerinbestimmung aufbauen kann. 